(C) [2006] IEEE. Reprinted, with permission, from [Wenjing Jia, Gaussian Weighted Histogram Intersection for License Plate Classification, Pattern Recognition, 2006. ICPR 2006. 18th International Conference on (Volume:3 ), 2006] . This material is posted here with permission of the IEEE. Such permission of the IEEE does not in any way imply IEEE endorsement of any of the University of Technology, Sydney's products or services. Internal or personal use of this material is permitted. However, permission to reprint/republish this material for advertising or promotional purposes or for creating new collective works for resale or redistribution must be obtained from the IEEE by writing to pubs-permissions@ieee.org. By choosing to view this document, you agree to all provisions of the copyright laws protecting it 


\title{
Gaussian Weighted Histogram Intersection for License Plate Classification
}

\author{
Wenjing Jia, Huaifeng Zhang, Xiangjian He, and Qiang $\mathrm{Wu}$ \\ Computer Vision Research Group, University of Technology, Sydney \\ \{wejia,hfzhang,sean,wuq\}@it.uts.edu.au
}

\begin{abstract}
The conventional histogram intersection (HI) algorithm computes the intersected section of the corresponding color histograms in order to measure the matching rate between two color images. Since this algorithm is strictly based on the matching between bins of identical colors, the final matching rate can be easily affected by color variation caused by various environment changes. In this paper, a Gaussian weighted histogram intersection (GWHI) algorithm is proposed to facilitate the histogram matching via taking into account matching of both identical and similar colors. The weight is determined by the distance between two colors. The algorithm is applied to license plate classification. Experimental results show that the proposed algorithm produces a much lower intra-class distance and a much higher inter-class distance than previous HI algorithms for tested images which are captured under various illumination conditions.
\end{abstract}

\section{Introduction}

Using color histogram as a stable representation over change in view for object recognition was explored by Swain and Ballard [1][2]. They introduced the color indexing technique to efficiently recognize objects by matching their color histograms using histogram intersection (HI) method. This algorithm did not address the issue of illumination variation.

Towards an illumination-insensitive histogrambased image matching algorithm, many alternations have been suggested. These methods can be divided into two groups. The first group, instead of using color directly, aims to generate histograms from other features. This includes Funt et al.'s [3] ratio of color RGB triples, and Nayar et al.'s [4] color reflection ratios. Gevers et al. [5], Jia et al. [6], and Zhang et al. [7] further developed the color ratio gradient to make it insensitive to the geometry and position change of the object, shadows, illuminations, and other imaging conditions. The second group tries to improve the defini- tion of histogram intersection. Wong et al. [8] proposed a merged palette histogram matching (MPHM) method. Using thee method, two perceptually similar colors, rather than identical colors, are able to be intersected.

The idea proposed in the paper belongs to the second group. Note that, in the MPHM method, the weight, which describes the contribution of a set of similar colors used to matching the given color, is the same. This, however, does not well reflect the matching contributed by different colors. In this paper, a Gaussian weighted histogram intersection (GWHI) algorithm is proposed, where a weight function is used to differentiate the contributions by different colors.

The proposed GWHI algorithm is applied to license plate classification. During our research on automatic license plate recognition [9], it is found that there are different classes of license plates with quite different features. Our aim is to differentiate license plates which belong to different classes for the benefit of further recognition. Due to variant environment under which the license plate images were taken, there are unavoidably big variations between license plates of same classes in their color. A robust feature and matching method is hence needed.

The remaining parts of the paper are organized as follows. The conventional HI method is introduced in Sect. 2. In Sect. 3, Gaussian weighted HI is illustrated in details. In Sect. 4, the proposed algorithm is tested and the results are compared with the conventional HI and the MPHM. Conclusions are given in Sect. 5.

\section{Conventional histogram intersection}

Histogram-based image retrieval algorithms try to measure the similarity in contents via their histograms between a model image and any images in database, i.e., target images, in order to properly classify or retrieve images. Histogram intersection (HI), proposed by Swain and Ballard [1][2], is a straightforward method to calculate the matching rate between two histograms for this purpose. 


\subsection{Histogram intersection (HI)}

Assuming the histograms of the model image and target image are $H_{M}$ and $H_{T}$ respectively, and each contains $n$ bins, Swain and Ballard [2] defined the intersection $H I$ of two histograms as:

$$
H I=\sum_{i=1}^{n} \min \left(h_{M}(i), h_{T}(i)\right)
$$

where both $H_{M}$ and $H_{T}$ are normalized by the total number of pixels in corresponding images.

It can be seen that the resultant fractional matching value between 0 and 1 is actually the proportion of pixels from the target image that have corresponding pixels of the same color in the model image. A higher matching rate indicates a higher similarity.

\subsection{Problems with existing HI algorithms}

The conventional $\mathrm{HI}$ algorithm has a limitation due to the fact that it assumes identical color matching, i.e., only corresponding bins of identical colors can be matched. In practice, however, the colors of real world images can be distorted both in the scene itself and in the image capturing process. Hence, images with same visual information but with slight color variation may degrade the similarity level significantly when the conventional $\mathrm{HI}$ is used.

In Fig. 1, two small "yellow" images are taken as an extreme example to illustrate this problem. The model image is a uniform image where all pixels take the color of $(R, G, B)=(230,215,50)$. Perceptually, a target image, as shown in the figure, is expected to give a good matching with this model image, even though there are slight color variations. As a matter of fact, however, the value for the corresponding bin of color $(230,215,50)$ in the target histogram is zero. Consequently, a null matching will be returned with the conventional $\mathrm{HI}$ method.

In order to overcome this problem, Wong et al. [8] proposed a merged palette histogram matching (MPHM) method. The essence of the method is to extend the intersection from bins of identical colors to bins of similar colors. In their algorithm, as long as the distance between two colors is less than a fixed threshold, the intersection between the bins of these two colors will be calculated. This algorithm has produced more robust image retrieval results for images captured under various illumination conditions. However, it assumes an identical weight of the contribution between colors which have different similarities with the given color. When applied to license plate image matching, the algorithm exhibited to be very sensitive to color variation. This is shown in Fig. 3(b), where the
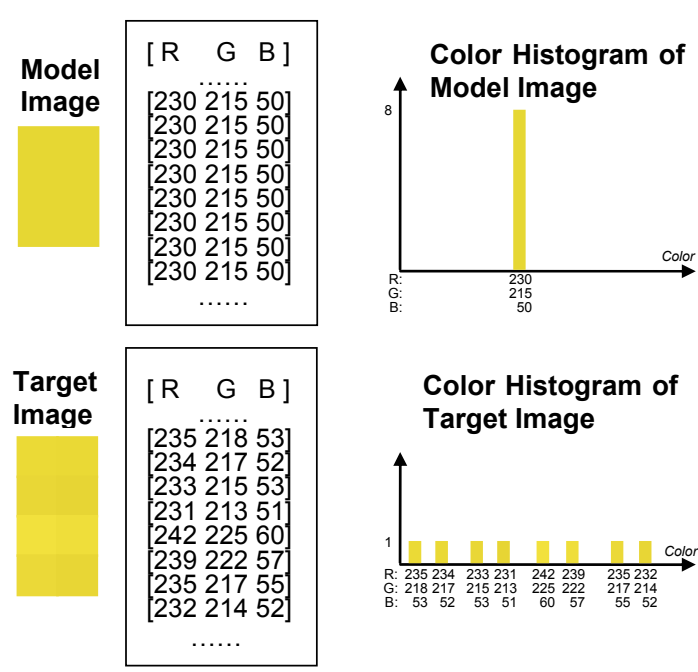

Color Histogram of Target Image

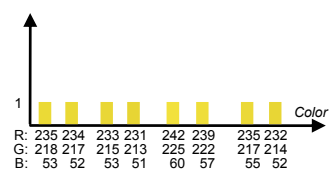

Fig. 1 An example of the problem with the conventional HI algorithm.

matching rates of same class of plate images vary greatly with the variation of colors.

In this paper, a Gaussian weight function is used to differentiate the contribution between colors which have different distance to the given color.

\section{Gaussian weighted HI (GWHI)}

Let us denote the distance between two colors $\vec{C}_{1}$ and $\vec{C}_{2}$ as $\left\|\vec{C}_{1}-\vec{C}_{2}\right\|$, and the weight as $w\left(\left\|\vec{C}_{1}-\vec{C}_{2}\right\|\right)$, which is a function of the distance between two colors. A generalized histogram intersection can be written as:

$$
H I=\sum_{i=1}^{n} \sum_{j=1}^{n} \min \left(h_{M}\left(\vec{C}_{i}\right), h_{T}\left(\vec{C}_{j}\right)\right) \cdot w\left(\left\|\vec{C}_{i}-\vec{C}_{j}\right\|\right)
$$

With this definition, the weight function in $\mathrm{HI}$ and MPHM algorithm corresponds to:

$$
w\left(\left\|\vec{C}_{i}-\vec{C}_{j}\right\|\right)=\left\{\begin{array}{cc}
1 & i f\left\|\vec{C}_{i}-\vec{C}_{j}\right\| \leq T h \\
0 & \text { otherwise }
\end{array}\right.
$$

where $T h$ is zero for the conventional HI algorithm and a non-zero constant for the MPHM algorithm.

A Gaussian function is used to describe the relationship between the color distance and the contribution as:

$$
w\left(\left\|\vec{C}_{i}-\vec{C}_{j}\right\|\right)=\left\{\begin{array}{cc}
f\left(\left\|\vec{C}_{i}-\vec{C}_{j}\right\|\right) & \text { if }\left\|\vec{C}_{i}-\vec{C}_{j}\right\| \leq B W \\
0 & \text { otherwise }
\end{array}\right.
$$

where $B W$ is the bandwidth of the weight function. The Gaussian function $f(x)$ in (4) is:

$$
f(x)=\frac{A}{\sqrt{2 \pi} \sigma} \exp \left(-\frac{x^{2}}{2 \sigma^{2}}\right) \quad-\infty<x<\infty
$$


In our practice, we take $99.9 \%$ energy to approximate the total energy in the infinite space and truncate the tail of the Gaussian function. Note that for a Gaussian function, when $x^{*}=3.3 \sigma$, we have:

$$
\int_{-x^{*}}^{x^{*}} f(x) d x=99.9 \% \int_{-\infty}^{\infty} f(x) d x
$$

So, the bandwidth of the Gaussian function in (4) is set as $B W=3.3 \sigma$.

The parameter $\sigma$ in (5) can be selected empirically. In order to compare the experimental results with the MPHM algorithm, we set the following restrictions on the selection of $\sigma$ :

(1) $f(x)_{\mid x=0}=1$;

(2) $\int_{-\infty}^{\infty} f(x) d x=2 T h$; and

(3) Using $99.9 \% \int_{-\infty}^{\infty} f(x) d x$ to approximate $\int_{-\infty}^{\infty} f(x) d x$. where $T h$ is the threshold set in the MPHM method. Condition (1) sets the contributions of identical colors in two methods as the same. Condition (2) and (3) makes sure the overall weights between two different methods are approximately same.

It can be worked out from above restrictions that, $\sigma \doteq 0.8 T h$ and $B W \doteq 2.64 T h$. The bandwidth in our experiment is set as $T h=15$, which is the same as that used in [8]. Thus, $\sigma=12$ and $B W=40$..

The definition of color distance is measured by the perceptual difference as:

$$
\left\|\vec{C}_{1}-\vec{C}_{2}\right\|=\sqrt{\left(l_{1}-l_{2}\right)^{2}+\left(u_{1}-u_{2}\right)^{2}+\left(v_{1}-v_{2}\right)^{2}}
$$

where $\vec{C}_{1}=\left(l_{1}, u_{1}, v_{1}\right)$ and $\vec{C}_{2}=\left(l_{2}, u_{2}, v_{2}\right)$ are two colors represented in CIEluv color space.

\section{Experiments}

\subsection{Experiment setup}

In this experiment, the GWHI algorithm is applied to vehicle license plate images. Our aim is to find a robust classification between different classes of license plate images. We say two license plate images belong to the same class when they have similar foreground and background colors, but they may have quite different contents (characters), size and viewing conditions. Two classes of license plates are frequently used in NSW, Australia. Without loss of generality, these two classes of vehicle license plate images are tested, namely, plates with yellow background and plates with white background.

We select a yellow license plate image as the model image. Then, the color histogram of the model image, in CIEluv color space, is computed and matched with those of total 91 various license plate images (target images), which belong to either same class or different classes. The model image and some examples of yellow and white target images are shown in Fig. 2. It can be seen that, due to various environment changes, images that belong to same class may have quite different appearance.

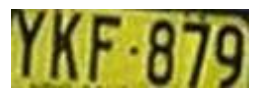

(a) Model image

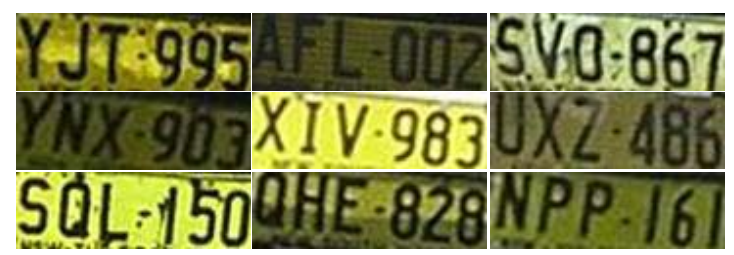

(b) 9 examples of yellow target images

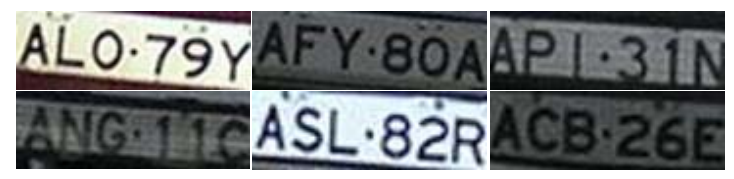

(c) 6 examples of white target images

Fig. 2 The model image and some examples of the yellow and white target images used in our experiments. All images are of $120 \times 40$ size.

\subsection{Experimental results and discussion}

The matching rates between the yellow model image and total 91 target images using the three HI algorithms mentioned in Sect. 3 are plotted in Fig. 3. Matching rates of yellow plates are painted in red dots and white plates in blue dots (darker if viewed in black-white picture).

As seen in Fig. 3(a) that, using the conventional HI, it is impossible to separate two classes of license plate images via their color histograms. Actually, even though the matching rates of white plates are very low $(\leq 0.17 \%)$, the matching rates of the tested yellow plates may also be very low. For instance, the matching rate of the yellow plate $\# 85$ is only $0.21 \%$.

Using the MPHM algorithm, this problem has been improved a lot. As shown in Fig. 3(b), the distance between the matching rates of two classes of plates has increased greatly. However, though using MPHM can separate two classes given that a proper threshold is carefully selected, the matching rates of same class license plates are still very sensitive to color variations. As demonstrated, the data of yellow images still spread in a wide range (from $31.65 \%$ to $99.73 \%$ ). 
Using the proposed GWHI algorithm, it can be seen from Fig. 3(c) that, matching rates of both same class and different classes of plates have increased. This is due to the fact that a wider bandwidth has been used than that in the MPHM algorithm. At the same time, the matching rates of same class license plates are much more stable compared to the results of using $\mathrm{HI}$ and MPHM algorithm. There are 47 of $62(75.8 \%)$ yellow plates with a matching rate larger than $90 \%$ and only 5 of $62(8 \%)$ yellow plates with a matching rate less than $80 \%$. This is because we introduce a weight function rather than simply adding up all intersections contributed by different colors. Even though the matching rates between the yellow model and white target plates have increased at the same time, the distance between the matching rates of two classes is extended greatly.

\section{Conclusions}

In this paper, a new histogram intersection method, Gaussian weighted histogram intersection (GWHI), is proposed. A Gaussian function is used to weight the contribution to the matching by colors which have different distance with the given color. Our aim is to differentiate license plate images which belong to different classes. Experimental results show that using the proposed method, there are following benefits: (1) the intra-class distance becomes much smaller; and (2) the inter-class distance becomes much larger. In conclusion, the matching of color histogram using our GWHI method is less sensitive to color variation than previous HI methods.

\section{References}

[1] M.J. Swain and D.H. Ballard, "Indexing via color histograms", Proceedings of the Third International Conference on Computer Vision, 1990: pp. 390-393.

[2] M.J. Swain and D.H. Ballard, "Color Indexing", International Journal of Computer Vision, 1991. 7(1): pp. 11-32.

[3] B.V. Funt and G.D. Finlayson, "Color constant color indexing", IEEE Transactions on Pattern Analysis and Machine Intelligence, 1995. 17(5): pp. 522-529.

[4] S.K. Nayar and R.M. Bolle, "Reflectance ratio: A photometric invariant for object recognition", Proceedings of the Fourth International Conference on Computer Vision, 1993: pp. 280-285.

[5] T. Gevers and W.M. Smeulders, "Color constant ratio gradients for image segmentation and similarity of texture objects", Proceedings of the 2001 IEEE Computer Society Conference on Computer Vision and Pattern Recognition, 2001. 1: pp. I-18-I-25 vol.1.

[6] W. Jia, H. Zhang, X.. He, and Q. Wu, "Symmetric Colour Ratio in Spiral Architecture," Lecture Notes in Computer Science, Vol.3852, pp.204-213, 2006.

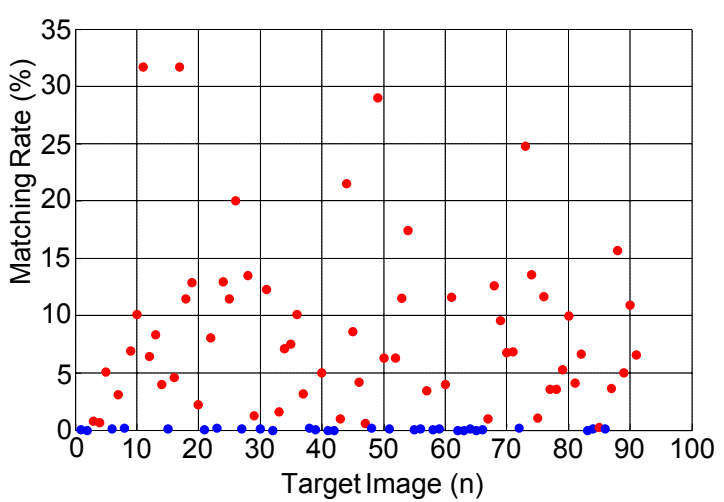

(a) Matching rates using conventional HI.

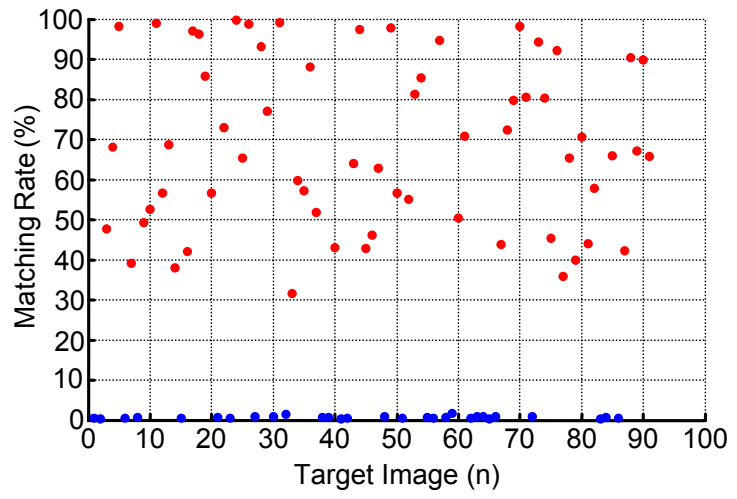

(b) Matching rates using MPHM.

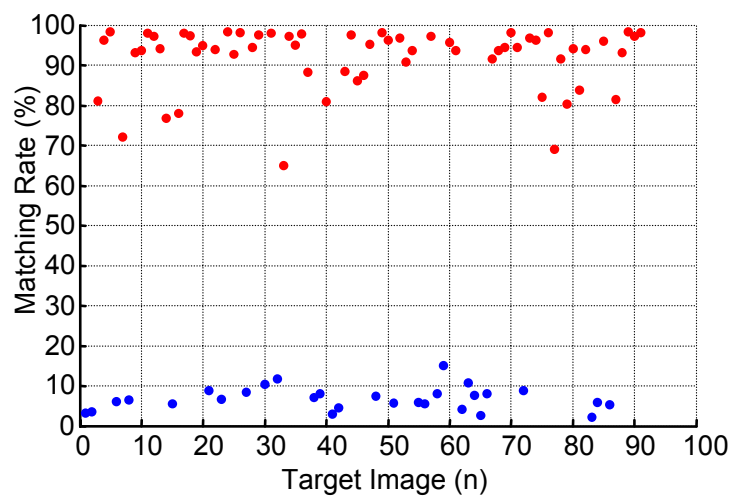

(c) Matching rates using GWHI.

Fig. 3 Histogram matching results using three HI algorithms.

[7] H. Zhang, W. Jia, X. He and Q. Wu, "Modified Colour Ratio Gradient," Proceedings of the International Workshop on Multimedia Signal Processing (IEEE), pp.317-320, 2005.

[8] K.-M. Wong, C.-H. Cheung, and L.-M. Po, "Mergedcolor histogram for color image retrieval," Proceedings of the 2002 International Conference on Image Processing, vol. 3, pp. 949-952 vol.3, 2002.

[9] W. Jia, H. Zhang, X. He and M. Piccardi, "Mean Shift for Accurate Number Plate Localization," Proceedings of the International IEEE Conference on Intelligent Transportation Systems, pp.566-571, 2005. 